Supporting Information for

\title{
Self-Healing of Nanoporous Gold Under Ambient
}

\section{Conditions}

Eun-Ji Gwak, ${ }^{\dagger}$ Hansol Jeon, ${ }^{\dagger}$ Eunji Song, ${ }^{\dagger}$ and Ju-Young Kim ${ }^{\dagger, *}$

${ }^{\dagger}$ School of Materials Science and Engineering, UNIST (Ulsan National Institute of Science and Technology), Ulsan 44919, Republic of Korea

*To whom correspondence should be addressed to J.-Y. Kim (juyoung@unist.ac.kr).

Experimental methods

Supporting Figures S1-S3

Caption of Supporting Movie 


\section{Experimental Methods}

Fabrication of np-Au samples and microstructure characterization: Np-Au samples were made by free-corrosion dealloying of precursor $\mathrm{Ag}_{70} \mathrm{Au}_{30}$ alloy prepared from $\mathrm{Ag}(99.99 \%)$ and $\mathrm{Au}(99.99 \%)$ pellets by melting at $1100^{\circ} \mathrm{C}$ and homogenization at $850^{\circ} \mathrm{C}$ under $\mathrm{N}_{2}$ atmosphere. The homogenized precursor alloy was cold-rolled to $1 \mathrm{~mm}$-thick plate, polished to $1 \mu \mathrm{m}$ with diamond suspension and annealed at $850^{\circ} \mathrm{C}$ for 24 hours to relieve stress and grain growth. The annealed precursor alloy was immersed into $35 \%$ nitric acid at room temperature for 5 days for free corrosion dealloying and repeatedly rinsed with ethanol and D.I. water. The Np-Au sample was examined by field emission scanning electron microscope (FE-SEM, FEI NovaNano 230) for imaging, energy-dispersive X-ray spectroscopy (EDS) to measure residual Ag, and electron backscatter diffraction (EBSD, TSL-OIM) to characterize grain structure.

Preparation of tensile specimen and tensile tests: Tensile specimens were prepared by using a focused ion beam (FIB, FEI Quanta 3D FEG) and Omniprobe system. Two- $\mu$ m-thick npAu leaf was machined using FIB, transferred and attached to a push-to-pull device (Hysitron). The attached np-Au leaves were machined to dog-bone shape with gauge length 3 $\mu \mathrm{m}$ and gauge width $2 \mu \mathrm{m}$ using FIB milling. The dimensions of gauge section of the tensile samples are $3 \mu \mathrm{m}$ length, $2 \mu \mathrm{m}$ width, and $2 \mu \mathrm{m}$ thickness, which includes 48000 cells in the gauge volume and 800 cells at the gauge cross-sectional area, approximately, where a cell is the summation of one ligament and one pore. In-situ tensile tests were conducted in SEM chamber (FEI Quanta 200 FE-SEM) and in-situ nanoindenter (Hysitron PI-87 Picoindeneter) with a diamond flat punch tip and strain rate of $4 \times 10^{-3} / \mathrm{s}$. Stress-strain curves are obtained after extracting stiffness of P-to-P device from raw force versus displacement curves. The 
strain distribution during tensile testing was analyzed using a digital image correlation (DIC) technique (Correlated Solutions, VIC-2D) from still images of recorded movies. After primary tensile tests, the tensile fractured samples were left alone under ambient conditions for 10 days in air at room temperature. After self-healing, the weldability of each ligament at the fracture surface was studied by strength recovery as tensile specimen were attached to Pto-P devices.

$3 D$ reconstruction: Specimens for $3 \mathrm{D}$ reconstruction were prepared as for tensile testing on the P-to-P device and its load and stiffness for primary tension and tension after self-healing for 10 days were measured using an in-situ nanoindenter in SEM chamber. Selected specimens of the same stiffness value before and after self-healing were infiltrated with epoxy into their porous structure and the top surface was protected by Pt coating. 3D reconstruction was carried out using a dual-beam focused ion milling system (FEI Helios Nanolab 650). Auto-slice and view program were used to cross-section and obtain SEM images and Avizo software (Avizo fire 8.1) was used to reconstruct 3D structure. Reconstructed volume is set to contain entire curved fracture surfaces with length-scale over $2 \mu \mathrm{m}$. The reconstructed volume can reflect actual deforming volume after self-healing, as shown in Figure 2f, and change of topological values. Connectivity, relative density, ligament size and ratio of fully connected ligaments to total ligaments were calculated using BoneJ plug-in of Image J and 'AnalyzeSkeleton' of FIJI software. Reconstructed volumes were set at over $100^{3}$ times of average ligament size. 


\section{Elastic modulus after self-healing}

It is important to note that strain values of initial tension and tension after self-healing were calculated with same standard of initial length, $l_{0}$, of gauge length. Since strain distribution is changed by initial fracture and self-healing, elastic modulus could become different by how we set $l_{0}$ for strain calculation as shown in Figure S3. Thus, the way we calculate strain value with initial length might cause overestimation of elastic modulus and fully recovered elastic modulus for some of self-healed specimens. As shown in Figure S3b and S3c, if we set initial length as $l_{0}$ ' of strain concentrated zone, elastic modulus will decrease from $5.810 \mathrm{GPa}$ to 2.911 GPa. If the elastic modulus decreases to $50.1 \%$, effective relative density will also decrease to $70.8 \%$ by the relation between elastic modulus, $E$, and relative density, $\rho_{\text {eff }}, \rho_{\text {eff }} \propto E^{1 / 2}$. [reference 29, Liu, L.-Z. et al., Acta Mater. (2016)]. 

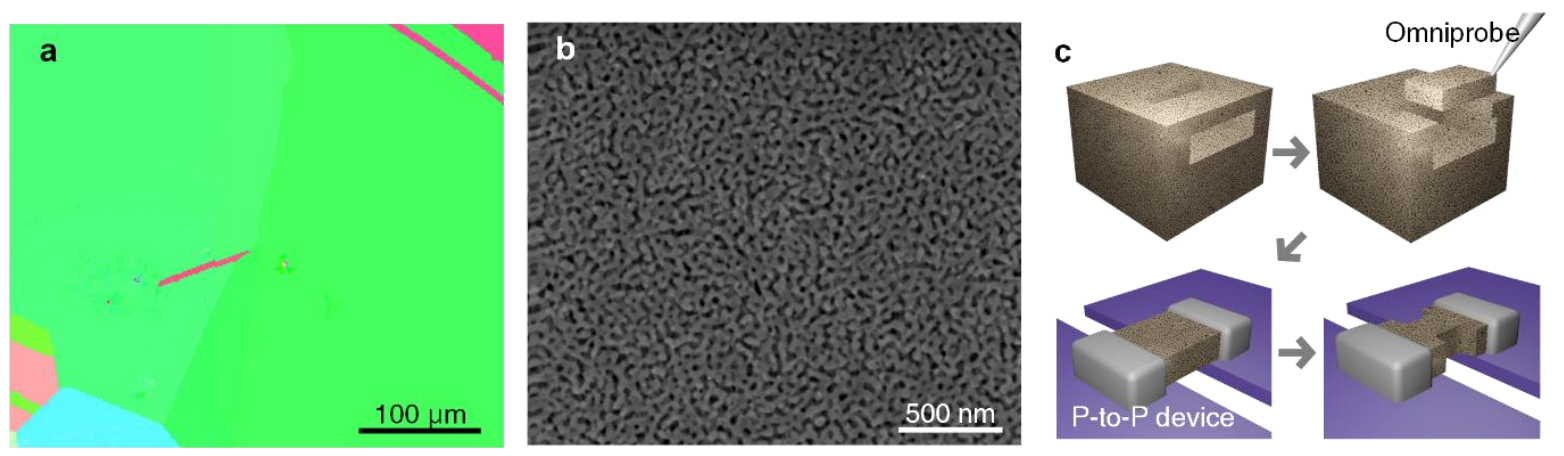

Figure S1. Microstructure of np-Au specimen and schematic figures of sample preparation for in-situ tensile test. a, Inverse pole figure image obtained from electron back-scattered diffraction (EBSD) and b, SEM image of surface on np-Au specimen. c, Dealloyed np-Au specimen is machined to a $2 \mu \mathrm{m}$-thick thin beam of np-Au by ion milling and lifted out using Omni-probe in FIB chamber. Lifted np-Au beam is attached on Push-to-Pull device and dogbone shape tensile specimen is fabricated. 
a

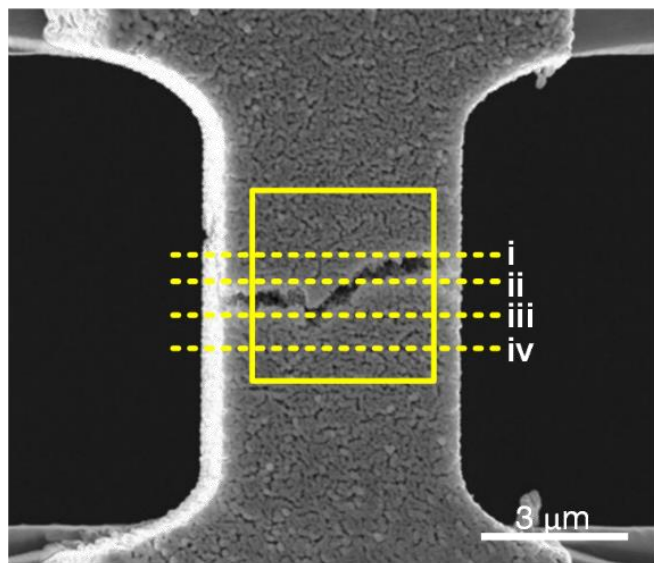

i

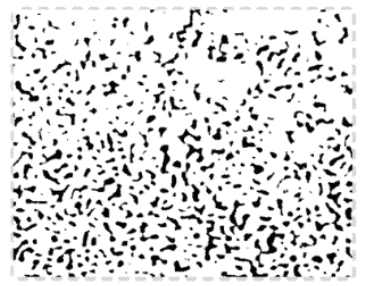

iii

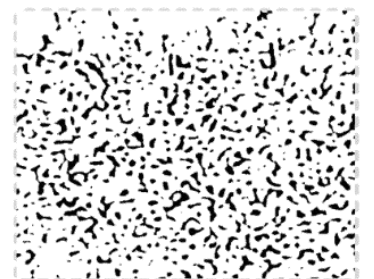

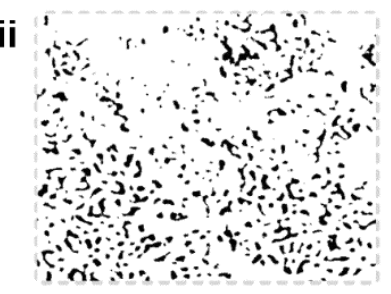

iv

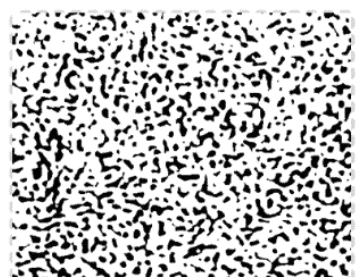

Figure S2. Cross-sectional image of np-Au specimen with curved fracture surface. a, SEM image of self-healed np-Au specimen. i-iv, Cross-sectional binary image of marked point in (a). Solid ligaments are colored in black and bi-continuous pores are colored in white. 

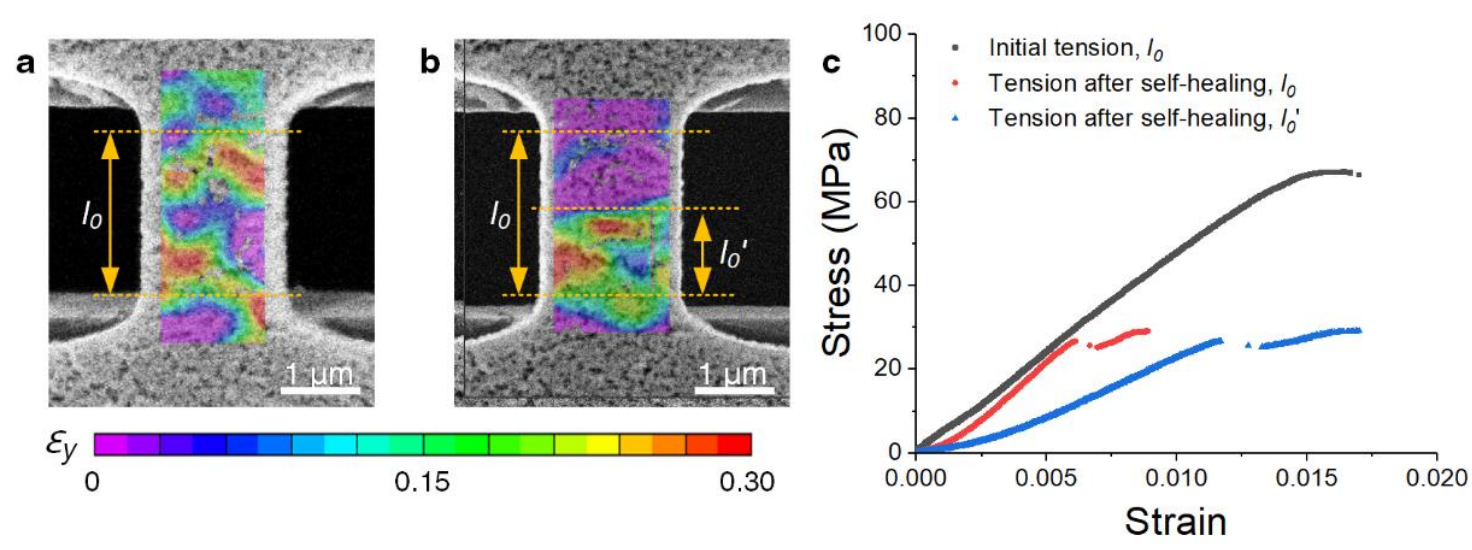

Figure S3. Change in elastic modulus by setting initial length to calculate strain. Strain distribution during a, initial tension and $b$, tension after self-healing. c, Stress-strain curves of initial tension, tension after self-healing calculated by changing initial length. $l_{0}$ is initial length of gauge length and $l_{0}$ ' is initial length of strain-concentrated region. 
a

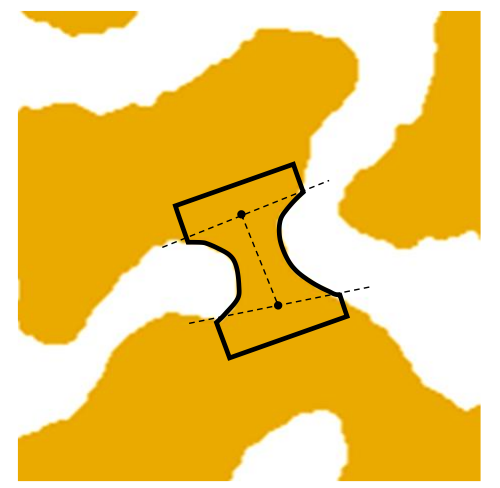

b

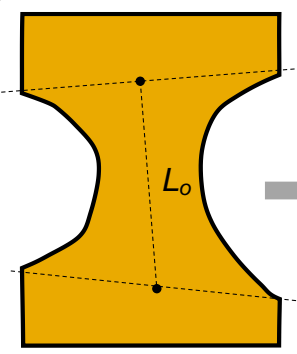

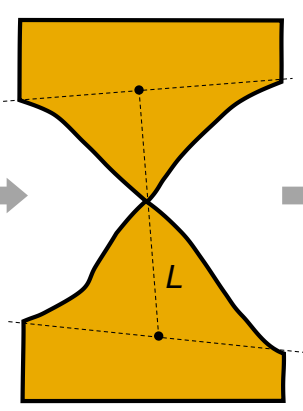

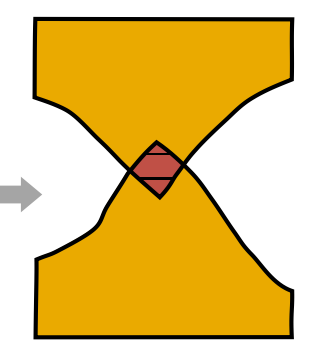

Figure S4. Schematic figures of head-to-head contact of failed ligaments. a, Binary image of ligament structure. b, Schematics of ligament when it is elongated by strain of $20 \%$ until failure and come back to original length. Elongation of ligament is defined by $\left(\mathrm{L}^{-} \mathrm{L}_{0}\right) / \mathrm{L}_{0}$ and marked region with red color indicates volume which should be contacted and compressed. 
Movie S1. In-situ tension movies with raw load versus displacement curves of initial tension, tension after self-healing for 1 day and 10 days in succession. 